\title{
La formación e-Learning: Un modelo conceptualmente útil para trasladar el Conocimiento a la Toma de Decisiones en Salud
}

\begin{abstract}
$E_{n}$ n nuestra sociedad actual de la información y el conocimiento, las organizaciones sanitarias públicas son paradigma de empresas de conocimiento, en las que el nivel de competencia de sus profesionales representa su máximo valor estratégico para hacer frente al cumplimiento de los objetivos de Atención a la Salud de los ciudadanos. Recientemente, la gestión del conocimiento a través de la Formación Continuada ( $\mathrm{FC}$ ) ha sido ampliamente reconocida como un elemento indispensable para la modernización de los Servicios de Salud y la mejora de la calidad y la seguridad en los procesos de atención; tal y como recoge el nuevo marco normativo desarrollado a partir de la Ley 44/2003 de Ordenación de las Profesiones Sanitarias, 55/2003 del Estatuto Marco y 16/2003 de Cohesión y Calidad del Sistema Nacional de Salud.
\end{abstract}

La FC es un proceso estructurado y planificado, dirigido a transformar la información en conocimiento, y el diseño de estrategias orientadas al entrenamiento de las competencias necesarias para el desempeño de las funciones que cada profesional tiene encomendadas. No existe, sin embargo, un cerrado consenso sobre cómo deben ser los modelos formativos que permitan salvar la conocida distancia entre "el saber y el hacer", acercando el conocimiento basado en la evidencia a la toma de decisiones en salud. Por el contrario, se ha demostrado que la mera recepción de conocimiento por parte de las personas que toman decisiones no implica que éstas lo utilicen, y que el conocimiento generado por las investigaciones en salud no son aplicados a la práctica clínica, sino a través de un sutil y largo proceso, en el que los fundamentos previos son gradualmente transformados.

Por otra parte, diversos estudios observacionales, desde Schön (1987) a Coles (1996), han fracasado a la hora de demostrar el impacto real de los modelos de formación académica tradicional en el desempeño de la práctica clínica de los profesionales sanitarios y/o de los resultados en salud. De esta forma, estos resultados vienen a cuestionar la eficacia de la FC tradicional, basada en exposiciones teóricas o clases magistrales junto a otras actividades más prácticas o participativas, a la hora de modificar la práctica profesional y mejorar los resultados en salud de la población.

Por el contrario, estos estudios sí encuentran cierta correlación entre los resultados alcanzados en la práctica clínica a largo plazo y la motivación de los profesionales por el perfeccionamiento de su competencia. Por ello, los autores concluyen que la FC actuaría más bien como fuente de motivación para la autoformación, así como un desencadenante del interés por actividades de Desarrollo Profesional Continuo (DPC), como puedan ser la revisión de la literatura y la consulta de fuentes de información científica y bases de datos, que probablemente serían las que inducirían un beneficio real a largo plazo.

Y es que el desarrollo de la competencia de los profesionales sanitarios depende de una serie de factores de gran complejidad, entre los que citaremos: nivel de conocimientos, habilidades técnicas, organizativas y de comunicación, capacidad de trabajo en equipo, buen juicio crítico y capacidad de razonamiento clínico para la resolución de problemas, conocimiento de las propias limitaciones, manejo de habilidades éticas, responsabilidad, etc. Pero, sobre todo, el factor que individualmente ha demostrado una mayor relevancia para aumentar la competencia profesional y la mejora del desempeño en la práctica clínica ha sido la adquisición y desarrollo de habilidades reflexivas, que le acompañarán a lo largo de toda su vida profesional. Éstas se basan en los postulados enunciados por Schön sobre el modo de aprendizaje de los profesionales, según los cuales la mayor fuente de conocimientos profesionales viene propiciada por la reflexión sobre la propia experiencia, realizada mejor de forma compartida (entre iguales), sobre el propio trabajo con los pacientes y los resultados obtenidos en salud. Conocer la evidencia técnica de lo que se debe hacer parece ser una condición necesaria, pero no suficiente, para asegurar un trabajo de calidad.

Este grado de complejidad ha determinado la evolución del énfasis desde la FC tradicional al más moderno término de DPC, que, además de englobar a la anterior, incorpora nuevos conceptos como procesos de reflexión individual y grupal, actividades de formación de carácter informal, consulta de fuentes de información científica, etc., que permitirán el desarrollo de un modelo de "resolución de problemas", el cual parece correlacionarse con una mayor eficacia. A través del conjunto de actividades incluidas como DPC, el profesional podrá alcanzar y mantener el nivel de competencia necesario a lo largo de toda su vida profesional, entendida ésta como la aptitud para integrar y aplicar conocimientos, habilidades y actitudes de gran complejidad asociados al ejercicio de su profesión, y que le permitirán desempeñar con éxito las exigencias de su puesto de trabajo en cada momento y situación. Todo ello con independencia del reconocimiento que, a través de la Carrera Profesional, pueda tener al ser aplicada con desigual acierto por las distintas organizaciones sanitarias. 
La consecuencia más importante que se deriva de todo lo comentado hasta el momento es que el proceso de reflexión crítica es el más importante a la hora de promover el cambio de actitudes y la modificación de conductas que permiten incorporar la evidencia científica para la mejora del desempeño en la atención sanitaria basada en el conocimiento.

A pesar de la relevancia del proceso de reflexión crítica, éste no siempre es suficientemente promovido por las propias organizaciones sanitarias durante la práctica profesional (en forma de sesiones clínicas, revisiones bibliográficas, de mortalidad, etc.), ni por el ambiente en el que se suelen desarrollar la mayor parte de las actividades de FC, incluso cuando éstas incorporan modernas metodologías docentes basadas en la realización de talleres de simulación, entrevista clínica, etc. Ello es debido a las dificultades para proporcionar un ambiente reflexivo y facilidades para la participación activa de todos y cada uno de los profesionales participantes, que tenga en cuenta los diferentes ritmos de aprendizaje, o a la premura de tiempo que dificulta la interacción.

Este proceso de reflexión crítica es, precisamente, el elemento diferencial más importante entre el tradicional concepto de FC y el más integral de DPC que permite integrar los conceptos de enseñanza de la FC con los de crecimiento profesional y personal, algo mucho más relacionado con la voluntad individual para el aprendizaje.

Podríamos concluir, por tanto, que es necesario diseñar estrategias formativas dirigidas a superar las dificultades encontradas para trasladar eficazmente a la práctica clínica el conocimiento científico generado por los resultados de la investigación en salud. Estas estrategias docentes deberán sobre todo basarse en el diseño de actividades que permitan ofrecer oportunidades para ejercitar el proceso de reflexión individual y grupal, mediante procedimientos como el análisis pormenorizado de resultados, la consulta de fuentes de información, el análisis comparado, la discusión en grupo o la revisión entre iguales (per-review). Todos ellos representan una parte consustancial a las actividades que incorporan los nuevos conceptos sobre el DPC.

EI DPC se configura así, cada vez más, como una dimensión fundamental para poder incorporar el conocimiento generado por la investigación en salud y mejorar la calidad de la atención en los Sistemas Sanitarios Públicos. Éste facilitará un avance progresivo desde la formación inicial hasta los diversos estadios de profesional competente que permita finalmente alcanzar el nivel de experto o de excelencia profesional.

Es en este contexto en el que es necesario enfatizar la utilidad comprobada, ya desde hace algunos años en el mundo empresarial, de las nuevas metodologías de formación a distancia basadas en las nuevas tecnologías de la información y la comunicación. Nuevas metodo- logías como la formación e-learning, distribuida a través de Internet, con la ayuda de sistemas de gestión del aprendizaje (Learning Management System), que permiten el control de todo el proceso de aprendizaje virtual que ha demostrado eficacia.

La aplicación de la formación e-learning al entorno de las ciencias de la salud, cuya utilidad no había sido testada en este ámbito hasta el momento, posibilita el diseño de escenarios para replicar casos clínicos reales que permitan desplazarse por diversos itinerarios auto-formativos y participar en experiencias grupales, mediante la distribución de contenidos y diversas herramientas de comunicación en un mismo espacio virtual. Escenarios que proporcionan oportunidades para ejercitar el proceso de reflexión, mucho más difícil de conseguir con las actividades formativas presenciales tradicionales.

Oportunidades que la formación e-learning sí puede proporcionar más fácilmente a través de una más reflexiva y prolongada interacción de los participantes con las fuentes de información científica y bases de datos seleccionadas, la utilización de contenidos adaptados al caso, así como la posibilidad de utilizar dinámicas de grupo dirigidas a compartir conocimiento y experiencias, en un proceso que permite la participación de todos, y que podrá ser tutelado y dinamizado por expertos facilitadores del proceso de aprendizaje.

Un modelo de formación que permite recrear algunos de los elementos más importantes de la "formación en contexto", y que resultará de gran utilidad para facilitar la interacción y el intercambio de información y experiencias entre los profesionales sanitarios. Todo ello junto a una combinación de diversas modalidades educativas que utilizan metodologías activas que dan un mayor protagonismo al profesional a la hora de seleccionar el itinerario formativo, la planificación de las actividades formativas adaptada a sus necesidades, proporcionan un mayor grado de autonomía y claras ventajas para promover la modificación de conductas en la práctica clínica profesional.

Este modelo no excluye la programación combinada de actividades formativas presenciales (blended learning) para reforzar el compromiso y ensayar las habilidades técnicas que no se pueden adquirir a distancia, lo que resultará extraordinariamente eficaz para limitar al mínimo de tiempo posible la dedicación y esfuerzo por parte del profesional. Se facilita así el acceso a actividades de DPC con mayor grado de independencia del tiempo y lugar en el que el profesional desempeñe su actividad, actuando de esta forma sobre el coste del "factor tiempo", que ha demostrado ser una de las mayores limitaciones a la expansión de los procesos de DPC.

La formación e-learning ha sido ensayada con éxito en los últimos años por el Servicio de Salud de Castilla-La 
Mancha (SESCAM), e incorporada en toda la organización como una parte del proceso de modernización dirigida a la gestión integral del conocimiento. Los excelentes resultados alcanzados han situado al SESCAM en una posición de liderazgo en este campo dentro del Sistema Nacional de Salud, constituyendo un referente para otros Servicios de Salud. Así, la evaluación de los resultados alcanzados por la formación e-learning, y en particular por el "Programa de Formación en la Atención a Patologías más Prevalentes y el Uso Racional del Medicamento", en el que han participado ya nada menos que 3.110 licenciados sanitarios, lo que por si mismo supone un hito sin precedentes, ha demostrado elevados grados de satisfacción y buenos resultados en los indicadores intermedios del proceso docente de estos profesionales.

Por otra parte, algunos datos preliminares disponibles sugieren la existencia de una positiva correlación del grupo de profesionales participantes en este programa e-learning con la mejora de la calidad de la prescripción y el impacto real en el uso racional del medicamento (datos no publicados), apoyando lo comentado en el presente artículo bajo el punto de vista conceptual.

Será necesario realizar posteriores estudios controlados que permitan establecer los beneficios de este modelo de formación a la hora de facilitar la modificación de conductas, dirigidas a la incorporación de la medicina basada en pruebas de evidencia en la práctica clínica y la mejora de los resultados en salud. Ello permitirá establecer la utilidad del modelo para disminuir el intervalo entre la producción de conocimiento generado por los resultados de la investigación en salud y su incorporación a la práctica de la atención sanitaria basada en el conocimiento.

José Antonio Cantalapiedra Santiago Coordinador de Investigación, Docencia y Formación

Servicio de Salud de Castilla-La Mancha

\section{BIBLIOGRAFÍA}

1. Ruiz de Ardana R. La Formación como elemento de mejora de la calidad en las Organizaciones Sanitarias. Sedisa 2007;(Dic):32-41.

2. Ley $44 / 2003$ de 21 de noviembre, "de Ordenación de las Profesiones Sanitarias". BOE de 22 de noviembre de 2003; 280:41442-58.

3. Oriol Bosch A, Roma Millán J. El desarrollo profesional continuo en el contexto del nuevo profesionalismo. JANO 2006; 1626:14-18.

4. Traducción del Conocimiento (Editorial). Avances en el Desarrollo Profesional Continuo en Medicina. DPM 2008; 1(1):3-5.

5. Rosenberg MJ. E-Learning: Strategies for Delivering Knowledge in the Digital Age. London: McGraw-Hill Trade; 2000.

6. Pardell Alentá H, Pallarés Nelia L, Segura Badia H. De la formación médica continuada al desarrollo profesional continuo. JANO 2006; 1626:30-33.

7. Maillet B. Retos de la Formación Médica Continuada. Educ Méd 2006; 9:173-180.

8. Lobos JM. Guerra G. Papel de las Sociedades Científicas en el Desarrollo Profesional Continuo del Médico. Avances en el Desarrollo Profesional Continuo en Medicina. DPM 2008; 1(1):712.

9. Pallarés Nelia L, Pardell Alentá H. Retos de la Formación Médica Continuada. Situación actual y perspectivas de futuro en España. Educ Méd 2006; 9:180-183.

10. Davis DA, Evans M, Jadad A, Tugwell P. The case for knowledge translation: shortening the journey from evidence to effect. BMJ 2003; 327:33-35.

11. Bruguera Cortada M. Gual Sala A. Retos del Desarrollo Profesional Continuado y la Regulación de la Profesión Médica. Educ Méd 2006; 9: 193-200. 